\title{
A Piezoelectric Flexible Insole System for Gait Monitoring for the Internet of Health Things
}

\author{
Junliang Chen ${ }^{1}$, Yanning Dai ${ }^{1}$ and Shuo Gao ${ }^{1,2, *}$ \\ ${ }^{1}$ School of Instrumentation and Optoelectronic Engineering, Beihang University, Beijing 100191, China. \\ ${ }^{2}$ Beijing Advanced Innovation Center for Big Data-Based Precision Medicine, Interdisciplinary Innovation Institute of Medicine \\ and Engineering, Beihang University, Beijing, 100191, China \\ *shuo_gao@buaa.edu.cn
}

\begin{abstract}
Gait analysis is important in the field of healthcare, due to its close relationship to chronic diseases. With the development of the Internet of Health Things (IoHT), long-term gait monitoring and corresponding analysis can be performed remotely, reducing a patient's time and traffic cost, while providing doctors more valuable gait information. In this paper, we present a piezoelectric insole gait monitoring system and its use in an IoHT architecture. Through the experimental results, the high detection sensitivity of $54 \mathrm{mN}$ and responsivity of $163 \mathrm{mV} / \mathrm{N}$ are achieved, thereby satisfying the need for analyzing various diseases. Furthermore, the assembled system can continuously work for 16 hours, indicating its successful utilization when longterm gait monitoring is required. The presented work provides a feasible means for real-time, long-term, and accurate gait monitoring, prompting the development of gait analysis in the IoHT.
\end{abstract}

Keywords-Piezoelectric force sensing; flexible insole device; gait monitoring; Internet of Health Things

\section{INTRODUCTION}

Gait is closely associated with many chronic diseases, as well as their progressions (e.g., stroke [1-2], tarsal coalition [3], Parkinson's disease [4-5], and diabetes [6-7]). It is of great significance to monitor gait parameters, such as velocity [8], stride length [9], and plantar pressure distribution accurately and continuously for disease evaluation [4] and efficient rehabilitation [10]. With the emergence of the Internet of Health Things (IoHT), real-time, long-term, and remote gait monitoring has been obtained, enabling a more personalized, preventive, and collaborative form of care for patients [11].

Some recent studies have demonstrated the use of gait monitoring in IoHT scenarios. In [12], five force sensitive resistor (FSR) sensors were used within an insole to count steps accurately under five walking styles (normal walking, jogging, sprint, star ascent, and stair descent), thus providing emergency detection (e.g., falling) for senior people. In [13], a wireless insole system was developed using the four FSRs to analyze how the patient's pressure distribution is affected by abnormalities, fractures, etc. In [6], an insole optical fiber sensor was presented for continuous and remote gait pressure distribution monitoring and to evaluate the evolution of ulcerations for patients of diabetes. However, there are still some challenges that need to be addressed. First, the limited number of sensing points places a restriction on gait recovery resolution [6, 12-13]. Second, these IoHT gait analysis systems are based on active sensing techniques [6, 12-13], which suffer from high energy consumption, thus limiting their successful use in IoHT architecture.

To resolve the above issues, in this work, a flexible piezoelectric based insole is implemented to measure a patient's plantar perpendicular pressure. The 24 sensing points ensure a high 2-dimensional space resolution, and flexible piezoelectric polyvinylidene fluoride (PVDF) film makes the insole sensor suitable for frequent bending during walking. The intrinsic mechanical-to-electrical conversion ability of the piezoelectric effect passively detects force signals, potentially extending the battery's lifetime. The system is integrated with wireless communication functions, allowing the data to be sent to remote end terminals. The use of the presented technique in an IoHT scenario is explained at the end of the article.

\section{METHODOLOGY}

\section{A. Sensor Fabrication}

The structure of the gait sensing insole device and the assembled system is shown in Fig. 1 (a) and (b). Commercial PVDF film (Solvay) was placed in the middle as force sensing layer. 24 square copper electrodes (side length $4 \mathrm{~mm}$ ) etched on a polyimide substrate were placed on top of the force sensing layer. In ground electrode layer, another 24 electrodes connected together were also etched on a polyimide substrate. These three layers formed a $\mathrm{Cu} / \mathrm{PVDF} / \mathrm{Cu}$ sandwiched piezoelectric architecture.

In the outermost layers, two polyethylene terephthalate (PET) films were used to protect the piezoelectric insole device. A back view photograph of the right foot insole device is shown in Fig. 1 (c). The different layers are laminated by the technique presented in [14].

\section{B. System Construction}

The fabricated insole device is connected to readout circuitry and the signal is processed and wirelessly delivered via the 


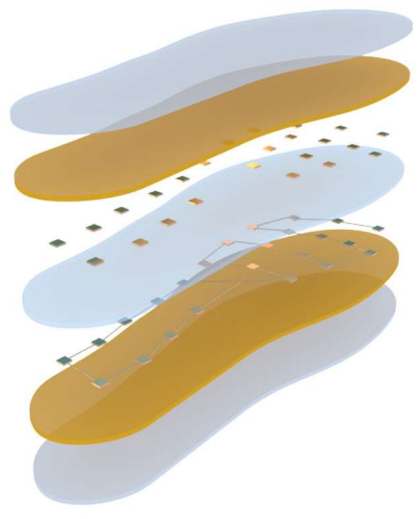

\begin{tabular}{|c|}
\hline PET: $120 \mu \mathrm{m}$ \\
\hline Polyimide: $50 \mu \mathrm{m}$ \\
\hline Top Electrodes: $1 \mu \mathrm{m}$ \\
\hline PVDF: $52 \mu \mathrm{m}$ \\
\hline GND Electrode: $1 \mu \mathrm{m}$ \\
\hline Polyimide: $50 \mu \mathrm{m}$ \\
\hline PET: $120 \mu \mathrm{m}$ \\
\hline
\end{tabular}

(a)

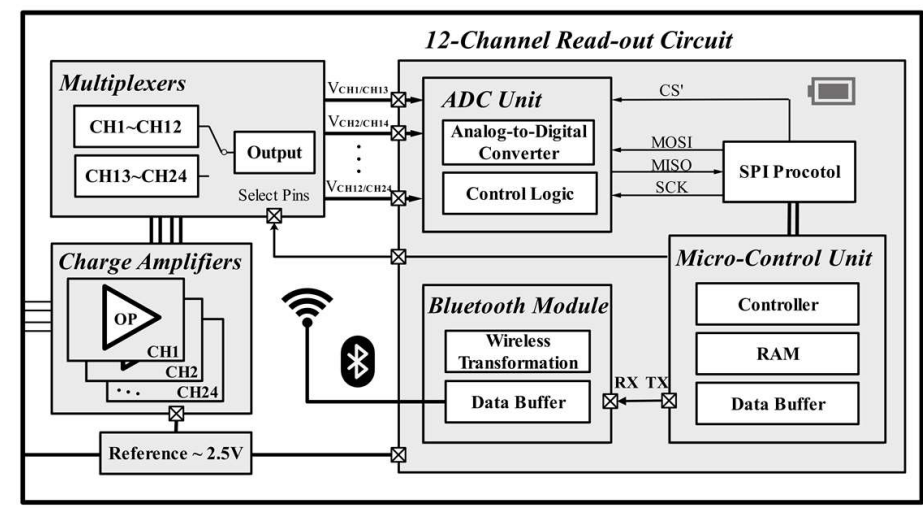

(d)

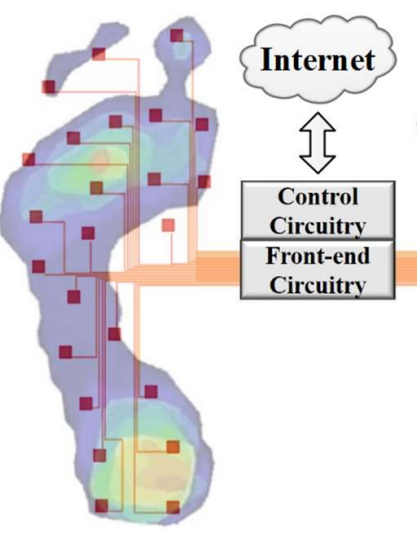

(b)

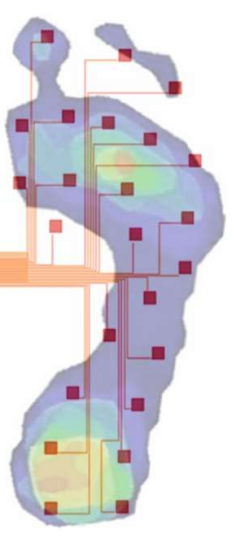

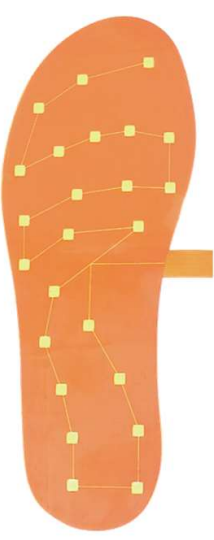

(c)

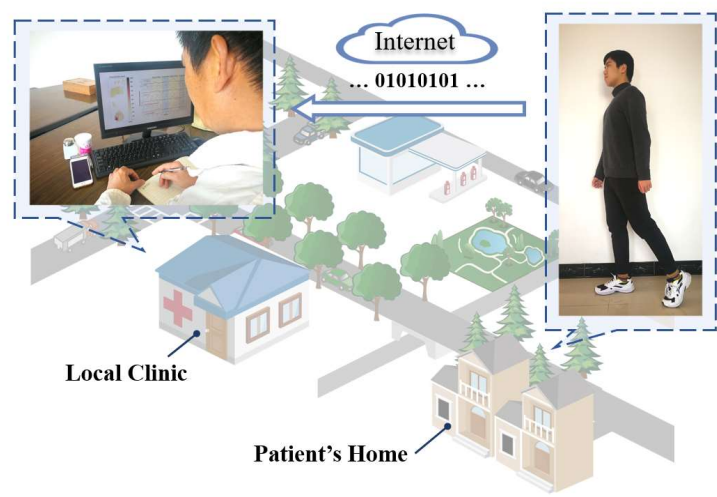

(e)

Fig. 1 (a) The structure of piezoelectric based insole sensor; (b) Conceptual diagram of gait monitoring system; (c) Back view photograph of right foot insole force sensing device; (d) The block diagram of circuitry for detecting, processing and transmitting force induced charges during walking; (e) Remote gait monitoring between patient's home and local clinic.

TABLE I. SUMMARY OF THE MAJOR CIRCUIT PARAMETERS

\begin{tabular}{|c|c|}
\hline \multicolumn{2}{|c|}{ Circuit Parameters } \\
\hline Input Dynamic Range & $0 \mathrm{~V}$ to $+5 \mathrm{~V}$ \\
\hline ADC Resolution & $12 \mathrm{Bit}$ \\
\hline Data Rate & $230,400 \mathrm{bps}$ \\
\hline Scanning Rate & $150 \mathrm{~Hz}$ \\
\hline Battery Capacity & $800 \mathrm{mAh}$ \\
\hline Working Current & $50 \mathrm{~mA}$ \\
\hline
\end{tabular}

Internet. Fig. 1 (d) presents a block diagram of the readout and communication circuitry. The reference voltage $(2.5 \mathrm{~V})$, provided by the DC-DC voltage regulator, AMS1117, is attached to the insole ground layer and the positive input of the amplifiers to ensure the single power supply's working conditions. The generated charges during the walking event are transferred to voltage signals at charge amplifiers, and the output voltage floats up and down at a $2.5 \mathrm{~V}$ reference voltage. The multiplexers, TMUX1574 (2:1 4-channel switch, Texas Instruments), join the 24 amplifier outputs and a multichannel analog-to-digital convertor (ADC, ADS7952, 12 channels, 12bit SAR-ADC, Texas Instruments). After measuring the first 12 sensing point channel outputs $(\mathrm{CH} 1 \sim \mathrm{CH} 12)$, the micro-control unit (MCU, STM32F103C8T6, STMicroelectronics) changes the logical voltage of the multiplexer select pins, and then the multiplexers join another 12 sensing points channel outputs (CH12 CH24) to the ADC. At the same time, the digital output of the ADC is conveyed to the MCU random access memory (RAM) through a serial peripheral interface (SPI) protocol for the buffer. These collected data can be transmitted wirelessly to physically disconnected terminals like computers and mobile phones for storage and further processing via a Bluetooth module (HC-42, Huicheng). Hence, the patients' gait information can be collected at home and transmitted to remote doctor terminals, as shown in Fig. 1 (e). Considering that the step frequency is between $0.7 \mathrm{~Hz}$ to $1.51 \mathrm{~Hz}$ [15], the scanning rate of the whole 24 sensing points is $200 \mathrm{~Hz}$ at a 230,400 bps data rate, thereby satisfying the sampling rate specifications required for the gait analysis and accurately recovering the gait during walking [15]. The system's circuit performance is given in Table I.

\section{RESULTS AND DisCUSSION}

\section{A. Device Performance}

For force sensing devices, force detection sensitivity and force-voltage responsivity are two important parameters, which are examined in this section. The results of 24 sensing points of a pair of insoles are given in Fig. 2 (a). The average sensitivity and responsivity of the 24 sensing points are $54 \mathrm{mN}$ and 163 


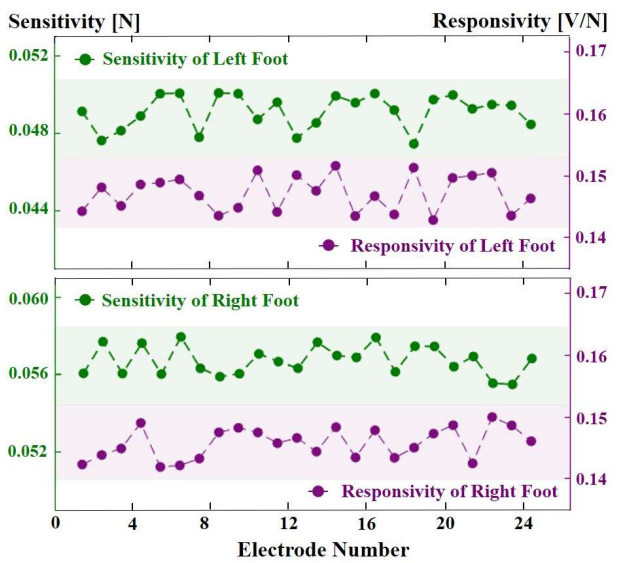

(a)

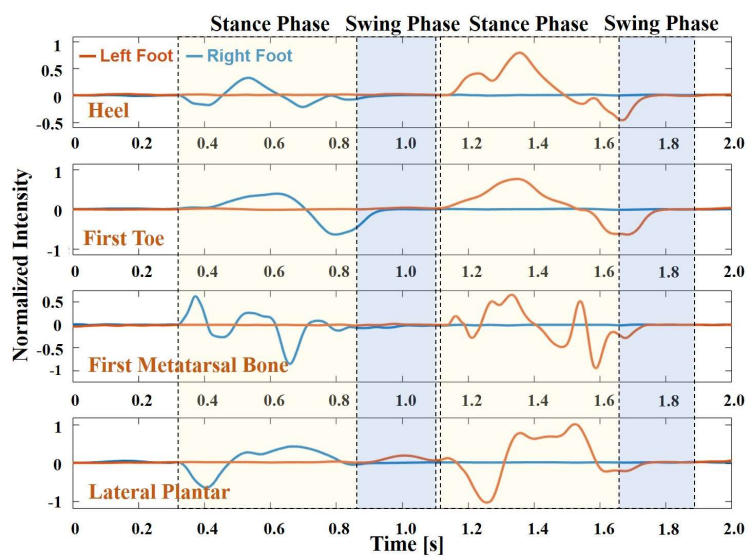

(b)

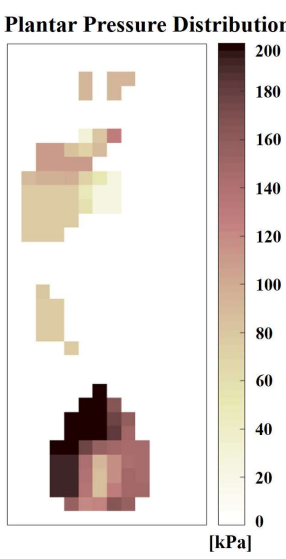

(c)

Fig. 2 (a) Sensitivity and responsivity of the whole 24 sensing points at different feet; (b) Normalized pressure intensity monitoring of four typical sensing points during walking; (c) 2D plantar pressure distribution of left foot.

TABLE II. QUANTIFIED SPECIFICATIONS FOR GAIT ANALYSIS. SOURCE: COMMERCIAL PRODUCTS AND THE REPORTED RESEARCHES [3, $6,16-18]$

\begin{tabular}{|c|c|c|}
\hline \multicolumn{3}{|c|}{ Quantified Specifications for Gait Analysis } \\
\hline Parameters & Required & Achieved \\
\hline $\begin{array}{c}\text { Perpendicular Pressure Dynamic } \\
\text { Range for Single Electrode }\end{array}$ & $260 \mathrm{kPa}$ & $300 \mathrm{kPa}$ \\
\hline Normal Stress Sensitivity & $100 \mathrm{mN}$ & $54 \mathrm{mN}$ \\
\hline Scanning Rate & $>60 \mathrm{~Hz}$ & $150 \mathrm{~Hz}$ \\
\hline $\begin{array}{c}\text { Temporal Resolution of } \\
\text { Cycle Duration }\end{array}$ & $\leq 10 \mathrm{~ms}$ & $6.7 \mathrm{~ms}$ \\
\hline
\end{tabular}

$\mathrm{mV} / \mathrm{N}$, respectively. Compared to the quantified specifications (given in Table II) needed for medical gait analysis, satisfactory device performance has been achieved for the purpose of disease diagnosis, such as diagnosis of tarsal coalition [3], Parkinson's disease [4-5], and diabetes [6-7].

\section{B. Experimental Results of Gait Monitoring}

The pressure monitoring of four typical sensing points (first toe, heel, first metatarsal bone, and lateral plantar [5]) from two feet in a walking cycle is depicted in Fig. 2 (b). It can be observed that two important gait phases (stance and swing) are clearly demonstrated with corresponding pressure amplitude, proving the ability to diagnose diseases like stroke [1-2] and Parkinson's disease [4-5]. The 2-dimensional plantar pressure distribution shown in Fig. 2 (c) demonstrates the insole system's ability to provide detailed information for analyzing the development of diseases like Parkinson's disease [4-5].

As we explained previously, for wearable systems, battery lifetime is a significant consideration. Hence, the power consumption of the system was tested. As per the circuitry settings in Table I, a $50 \mathrm{~mA}$ current for one foot $(24$ sensing points) was detected, which means that a $800 \mathrm{mAh} 7.4 \mathrm{~V}(55$ $\mathrm{mm} \times 30 \mathrm{~mm} \times 11 \mathrm{~mm}, 35 \mathrm{~g})$ rechargeable lithium battery can supply the system for about 16 hours, allowing for a typical full day of gait monitoring [19]. The experimental results align with the results of the analysis.

In Fig. 1 (e), we also demonstrate this system's use for remote gait monitoring. The gait information of one subject at home was sent to the remote computer at a local clinic, indicating the feasibility of the developed insole gait monitoring system for the Internet of health things architecture.

\section{NOTES}

Ethics approval was gained from the associated clinic. Informed consent was obtained from each subject after a verbal and written explanation of the project.

\section{CONCLUSION}

In this work, an insole plantar pressure sensing system for the internet of health things (IoHT) is presented. The developed system can accurately detect and send subjects' gait information to a remote end terminal in a medical institution for disease analysis purposes. With the developed technique, users can avoid visiting hospitals frequently, while doctors can obtain more useful information from patients' daily activities. The presented technique offers convenience to both patients and doctors, thus advancing the development of the IoHT.

\section{REFERENCES}

[1] F. Moissenet, C. Bélaise, E. Piche, B. Michaud, and M. Begon, "An optimization method tracking EMG, ground reactions forces and marker trajectories for musculo-tendon forces estimation in equinus gait," Frontiers in neurorobotics, vol. 13, p. 48, 2019.

[2] F. B. van Meulen, D. Weenk, J. H. Buurke, B.-J. F. van Beijnum, and P. H. Veltink, "Ambulatory assessment of walking balance after stroke using instrumented shoes," Journal of neuroengineering and rehabilitation, vol. 13, no. 1, p. 48, 2016.

[3] A. Skwara, V. Zounta, C. O. Tibesku, S. Fuchs-Winkelmann, and D. Rosenbaum, "Plantar contact stress and gait analysis after resection of tarsal coalition," Acta Orthopcedica Belgica, vol. 75, no. 5, p. 654, 2009.

[4] B. Mariani, M. C. Jiménez, F. J. G. Vingerhoets and K. Aminian, "OnShoe Wearable Sensors for Gait and Turning Assessment of Patients With Parkinson's Disease," in IEEE Transactions on Biomedical Engineering, vol. 60 , no. 1 , pp. 155-158, Jan. 2013. 
[5] A. Achiron et al., "Primary progressive freezing gait," Movement disorders: official journal of the Movement Disorder Society, vol. 8, no. 3, pp. 293-297, 1993.

[6] M. F. Domingues et al., "Insole Optical Fiber Sensor Architecture for Remote Gait Analysis-An e-Health Solution," in IEEE Internet of Things Journal, vol. 6, no. 1, pp. 207-214, Feb. 2019.

[7] Z. Sawacha, G. Gabriella, G. Cristoferi, A. Guiotto, A. Avogaro, and C. Cobelli, "Diabetic gait and posture abnormalities: a biomechanical investigation through three dimensional gait analysis," Clinical biomechanics, vol. 24, no. 9, pp. 722-728, 2009.

[8] J.-J. Chou, C.-S. Shih, W.-D. Wang, and K.-C. Huang, "IoT sensing networks for gait velocity measurement," International Journal of Applied Mathematics and Computer Science, vol. 29, no. 2, pp. 245-259, 2019.

[9] A. J. A. Majumder, Y. ElSaadany, M. ElSaadany, D. R. Ucci, and F. Rahman, "A wireless iot system towards gait detection in stroke patients," in 2017 IEEE International Conference on Pervasive Computing and Communications Workshops (PerCom Workshops), 2017: IEEE, pp. 449454.

[10] G. Yang et al., "An IoT-enabled stroke rehabilitation system based on smart wearable armband and machine learning," IEEE journal of translational engineering in health and medicine, vol. 6, pp. 1-10, 2018.

[11] S. Pinto, J. Cabral, and T. Gomes, "We-care: An IoT-based health care system for elderly people," in 2017 IEEE International Conference on Industrial Technology (ICIT), 2017: IEEE, pp. 1378-1383.

[12] S. Siyang, T. Nilpanapan, and T. Kerdcharoen, "Development of IoTBased Data Shoes for Daily Life Step Count," in 2018 IEEE 7th Global Conference on Consumer Electronics (GCCE), 2018: IEEE, pp. 524-525.

[13] P. S. Malvade, A. K. Joshi, and S. P. Madhe, "IoT based monitoring of foot pressure using FSR sensor," in 2017 International Conference on Communication and Signal Processing (ICCSP), 2017: IEEE, pp. 06350639.

[14] Y Dai et al. "A Lammination Based Piezoelectric Insole Gait Analysis System for Massive Production for Internet of Things." International Journal of Distributed Sensor Networks, unpublished.

[15] S. Sprager and M. B. Juric, "Inertial sensor-based gait recognition: A review," Sensors, vol. 15, no. 9, pp. 22089-22127, 2015.

[16] A. Muro-De-La-Herran, B. Garcia-Zapirain, and A. Mendez-Zorrilla, "Gait analysis methods: An overview of wearable and non-wearable systems, highlighting clinical applications," Sensors, vol. 14, no. 2, pp. 3362-3394, 2014.

[17] R. G. Cutlip, C. Mancinelli, F. Huber, and J. DiPasquale, "Evaluation of an instrumented walkway for measurement of the kinematic parameters of gait," Gait \& posture, vol. 12, no. 2, pp. 134-138, 2000.

[18] A. Razak, A. Hadi, A. Zayegh, R. K. Begg, and Y. Wahab, "Foot plantar pressure measurement system: A review," Sensors, vol. 12, no. 7, pp. 9884-9912, 2012.

[19] S. Gill, J. Hearn, G. Powell, and E. Scheme, "Design of a multi-sensor IoT-enabled assistive device for discrete and deployable gait monitoring," in 2017 IEEE Healthcare Innovations and Point of Care Technologies (HI-POCT), 2017: IEEE, pp. 216-220. 